\title{
Tuning of Electrostatic vs. Depletion Interaction in Deciding the Phase Behavior of Nanoparticle-Polymer System

\author{
Sugam Kumar ${ }^{1 *}$, V. K. Aswal ${ }^{1}$ and J. Kohlbrecher ${ }^{2}$ \\ ${ }^{1}$ Solid State Physics Division, Bhabha Atomic Research Centre, Mumbai 400 085, India \\ ${ }^{2}$ Laboratory for Neutron Scattering, Paul Scherrer Institut, CH-5232 PSI Villigen, Switzerland \\ *E-mail:sugam@barc.gov.in
}

\begin{abstract}
Nanoparticle-polymer system interestingly show a re-entrant phase behavior where charge stabilized silica nanoparticles (phase I) undergo particle clustering (phase II) and then back to individual particles (phase I) as a function of polymer concentration. Such phase behavior arises as a result of dominance of various interactions (i) nanoparticlenanoparticle electrostatic repulsion (ii) polymer induced attractive depletion between nanoparticles and (iii) polymerpolymer repulsion, at different concentration regimes. Small-angle neutron scattering (SANS) has been used to study the evolution of interaction during this re-entrant phase behavior of nanoparticles by contrast-marching the polymer. The SANS data have been modeled using a two-Yukawa potential accounting for both attractive and repulsive parts of the interaction between nanoparticles. The degree of both of these parts has been separately tuned by varying the polymer concentration and ionic strength of the solution. Both of these parts are found to have long-range nature. At low polymer concentrations, the electrostatic repulsion dominates over the depletion attraction. The magnitude and the range of the depletion interaction increase with the polymer concentration leading to nanoparticle clustering. At higher polymer concentrations, the increased polymer-polymer repulsion reduces the strength of depletion leading to re-entrant phase behavior. The clusters formed under depletion attraction are found to have surface fractal morphology.
\end{abstract}

Keywords: Nanoparticle-polymer system, Depletion interaction, SANS.

PACS: 82.35.Np, 61.46.-w, 61.05.fg

\section{INTRODUCTION}

The colloidal dispersions have gained sufficient scientific interest as they are known to show rich phase behavior [1]. The phase behavior strongly depends on interparticle interaction which can be tuned by varying different solution conditions such as $\mathrm{pH}$, temperature, ionic strength etc. One of the model systems to understand these phase behavior is charge stabilized nanoparticles in the matrix of polymer. Apart from the scientific interests, these systems also have gained industrial attraction in the various fields such as biotechnology, optics, and electronics [2]. Presence of polymers in the solution of nanoparticles may either stabilize them through steric repulsion or destabilize by depletion attraction resulting from the adsorbing or non-adsorbing nature of polymers on nanoparticles, respectively. Further, it is also seen that even nonadsorbing polymers may re-stabilize nanoparticles at higher polymer concentrations in the presence of other factors like charge on polymer and/or non-ideal nature of the polymer [3]. Understanding of these effective intercations is the key to unlock the equilibrium phase behavior of these technically important systems.
We have observed an interesting re-entrant phase behavior of nanoparticles where the charged stabilized silica nanoparticles (phase I) undergo particle clustering (phase II) and back to individual nanoparticles (phase I) as a function of PEG polymer concentration. This phase behavior may arise as a result of cumulative effect of various interactions (i) repulsion between charged nanoparticles (ii) attraction between nanoparticles and polymer (iii) attraction between nanoparticles as caused by polymers (iv) polymer-polymer interaction, possibly present in the system. In this paper, we have performed small-angle neutron scattering (SANS) experiments to study the dominance of these interactions one over the other in order to explain this re-entrant phase behavior. SANS is an ideal technique to study such multi-component systems due to its unique advantage of easy possibility of contrast matching [4].

\section{EXPERIMENTAL}

Electrostatically stabilized colloidal suspensions of silica nanoparticles $($ size $=16.0 \mathrm{~nm})$ and PEG 
(radius of gyration $=5.6 \mathrm{~nm}, \mathrm{M} . \mathrm{W} .=6 \mathrm{~K}$ ) were purchased from Sigma-Aldrich. Samples were prepared by dissolving known amount of silica nanoparticles $(1 \mathrm{wt} \%)$ and varying PEG concentration in a mixed $\mathrm{H}_{2} \mathrm{O} / \mathrm{D}_{2} \mathrm{O}$ solvent $\left(15 \% \mathrm{D}_{2} \mathrm{O}\right)$ for which polymer is contrast-matched. Small-angle neutron scattering experiments were carried out at the SANS-I facility, Swiss Spallation Neutron Source SINQ, Paul Scherrer Institut, Switzerland [5]. The wavelength $(\lambda)$ of neutron beam used was $6 \AA$ with a $\Delta \lambda / \lambda$ about $10 \%$. Corrections were made for background and empty cell contributions and data were normalized to absolute cross-sectional unit using standard procedure.

\section{SANS ANALYSIS}

In SANS experiments, the coherent differential scattering cross section per unit volume $(\mathrm{d} \Sigma / \mathrm{d} \Omega)$ is measured as a function of wave vector transfer $[\mathrm{Q}=$ $(4 \pi \sin \theta) / \lambda ; 2 \theta$ is scattering angle]. For a mono-disperse particle system in a medium, it may be given by

$$
\frac{d \Sigma}{d \Omega}(Q)=n P(Q) S(Q)+B
$$

where $n$ is number density of particles. $P(Q)$ is intraparticle structure factor (square of the form factor) and $S(Q)$ is interparticle structure factor. $\mathrm{B}$ is a constant term representing incoherent background coming mainly from the hydrogen atoms present in the sample.

$\mathrm{P}(\mathrm{Q})$ is decided by the shape and size of the particle. For spherical particle of radius $R$, it may be written as [6]

$$
P(Q)=\frac{16 \pi^{2}}{9}\left(\rho_{p}-\rho_{s}\right)^{2} R^{6}\left[3 \frac{\sin (Q R)-(Q R) \cos (Q R)}{(Q R)^{3}}\right]^{2}
$$

where $\rho_{\mathrm{p}}$ and $\rho_{\mathrm{s}}$ are the scattering length densities of particle and solvent, respectively.

Interparticle structure factor $\mathrm{S}(\mathrm{Q})$ depends upon the spatial arrangement of the particles. It may be approximated to unity if the sample is dilute and effectively there is no particle-particle interaction at average distances. But in general, the interaction between the particles may be a combination of attractive and repulsive forces. In this case, S(Q) may be calculated by solving the Ornstein-Zernike (OZ) equation for following two-Yukawa (2Y) potential accounting both attraction as well as repulsion under Mean Spherical Approximation (MSA) closure relation [7].

$$
\begin{aligned}
U(r) & =\infty \text { for } 0<r<\sigma \\
& =-K_{1} \frac{\exp \left\{-\alpha_{1}(r / \sigma-1)\right\}}{r / \sigma}+K_{2} \frac{\exp \left\{-\alpha_{2}(r / \sigma-1)\right\}}{r / \sigma} \text { for } r>\sigma
\end{aligned}
$$

where $K$ (in units of $k_{B} T, k_{B}$ is Boltzmann constant and $T$ is temperature) is proportional to magnitude of the potential and $1 / \alpha$ is proportional to the range of the potential. The data have been analyzed by comparing the scattering from different models to the experimental data.

\section{RESULTS AND DISCUSSION}

Fig. 1 shows the SANS data of $1 \mathrm{wt} \%$ silica nanoparticles with varying PEG concentration $\left(\phi_{P}\right)$ in presence of $0.2 \mathrm{M} \mathrm{NaCl}$. Salt is added in order to reduce electrostatic repulsion between nanoparticles, so that depletion attraction driven clustering of nanoparticles may be observed. At low polymer concentration $(0.003 \mathrm{wt} \%)$, data shows low $\mathrm{Q}$ rise in the scattering intensity which increases on further increase in the polymer concentration $\left(\phi_{P}=0.01\right.$ and $0.1 \mathrm{wt} \%$ ) with the emergence of a Bragg peak at intermediate $\mathrm{Q}$ values. At still higher polymer concentrations $\left(\phi_{P}=1.0\right.$ and $\left.3.0 \mathrm{wt} \%\right)$, the Bragg peak disappears and the system shows a systematic fall in the low Q intensity. Finally the SANS data of $3 \mathrm{wt} \%$ polymer almost matches to that of pure nanoparticles reflecting the re-entrant behavior.

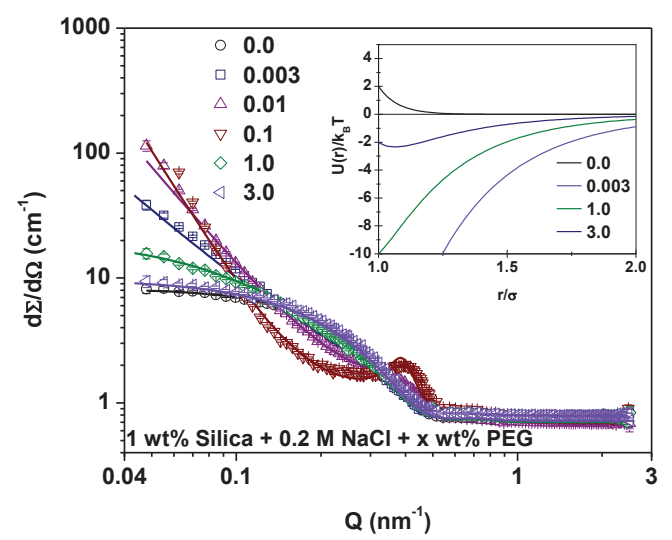

FIGURE 1. SANS data of 1 wt. \% silica nanoparticles with varying PEG- $6 \mathrm{~K}$ polymer concentration in presence of 0.2 $\mathrm{M} \mathrm{NaCl}$. Inset shows the fitted potentials.

The buildup of scattering at low polymer concentration suggests the presence of attractive interaction in the system [4]. The data have been analyzed by an $S(Q)$ as calculated for $2 \mathrm{Y}$ potential (eq.3) taking into account both attractive (depletion) and repulsive (electrostatic) parts of total interaction potential between nanoparticle. The parameters $\mathrm{K}_{2}$ and $\alpha_{2}$ corresponding to repulsion were kept fixed as calculated form concentrated pure nanoparticle solution whereas those $\left(\mathrm{K}_{1}\right.$ and $\left.\alpha_{1}\right)$ of depletion attraction were varied as fitting parameters. At low 
concentration $(<0.003 \mathrm{wt} \%)$, our analysis shows that range and strength of depletion attraction is more than that of electrostatic repulsion but the total interaction potential is less than $1.5 \mathrm{k}_{\mathrm{B}} \mathrm{T}$ at an average distance preventing nanoparticles from aggregation. On further addition of polymer, depletion attraction increases leading to nanoparticle clustering [3]. The linearity in the low Q data in log-log scale indicates the fractal nature of these clusters with ordered packing of particles within the aggregates as reflected in the Bragg peak observed around $Q_{p}=0.4 \mathrm{~nm}^{-1}$. The scattering from these clusters has been fitted by summing up the power law behavior of clusters and contribution from ordered particles within the clusters. These clusters are found large sized (order of micron) having surface fractal morphology with an average distance between the particles equal to particle diameter, suggesting the simple cubic packing particles within the clusters. The systematic decrease in the low $Q$ intensity for polymer concentration suggests the re-entrant of the initial phase. The SANS data at $\phi_{P}=1.0$ and $3.0 \mathrm{wt} \%$ have been again fitted by using $2 \mathrm{Y}$ potential. The strength and range both of depletion attraction first increases leading to the nanoparticle clustering and then decreases with increasing polymer concentration which gives rise to particle re-stabilization (inset of Fig. 1). Polymerpolymer repulsion is believed to be responsible for this reduction in depletion interaction at high polymer concentration [8]. At high polymer concentrations, the polymer chains do more work while transporting against a steep pressure gradient between depletion region and the bulk.

The re-entrant phase behavior of the nanoparticles in presence of polymer arises due to the interplay of the various interactions involved in the system. We have only varied the attractive interaction between the nanoparticles so far for a fixed repulsion. It will also be interesting to vary the repulsive part of the interaction for a fixed attraction. This is be simply done by varying the ionic strength of the $1 \mathrm{wt} \%$ nanoparticles and $1 \mathrm{wt} \%$ polymer system and the corresponding SANS data are shown in Fig. 3. Similar to the polymer case, the data shows increase in the low $Q$ intensity and the emergence of Bragg peak but no re-entrant of the initial phase is observed. The data in this set also has been analyzed by $2 \mathrm{Y}$ potential for salt concentrations $<0.2 \mathrm{M}$ [inset (a) of Fig. 3] whereas for nanoparticle clusters at higher salt concentrations. Though the system does not show any re-entrant phase behavior like that observed with polymers but the structure of salt induced aggregates has the same morphology as found with the polymers. It may also be mentioned that in the absence of PEG, salt alone is not enough to cause such attraction and aggregation [inset (b) of Fig. 3].

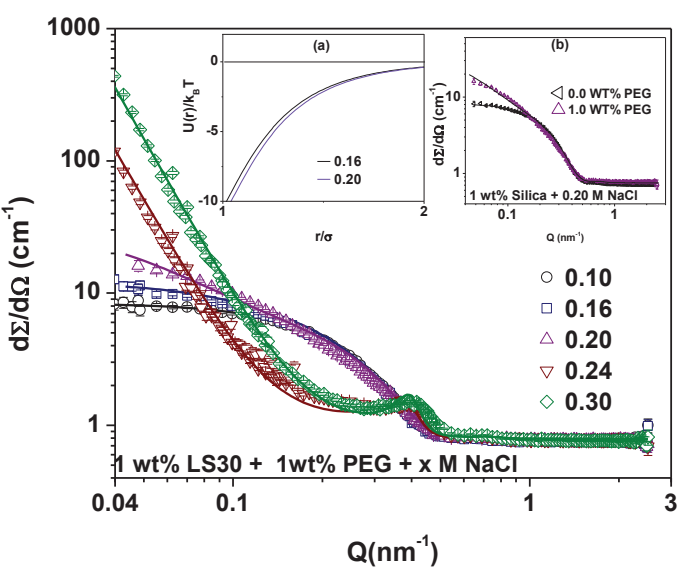

FIGURE 2. SANS data of $1 \mathrm{wt} \%$ of silica nanoparticles and PEG with varying salt concentration. Inset (a) shows the fitted potential whereas inset (b) shows the SANS data of 1 $\mathrm{wt} \%$ nanoparticles $+0.2 \mathrm{M}$ salt with and without $1 \mathrm{wt} \% \mathrm{PEG}$.

\section{CONCLUSION}

Role of electrostatic and depletion interaction were examined in silica nanoparticle and PEG polymer systems using small-angle neutron scattering. The system is found to show a re-entrant phase behavior where stable nanoparticles become unstable (particle clustering) and again re-stabilize with polymer concentration. Such phase transition has been explained in terms of electrostatic repulsion, attractive depletion between nanoparticles and repulsive polymer-polymer interaction. The total resultant potential has been modeled by two Yukawa potentials accounting for repulsive and attractive parts of the interaction. The phase behavior has been tuned by varying the degree of both of these parts (repulsive and attractive) through varying ionic strength and polymer concentration respectively.

\section{REFERENCES}

1. V. Anderson and H. Lekkerkerker, Nature 416, 811815 (2002).

2. G. Schmidt and M . M . Malwitz, Curr. Opin. Colloid Interface Sci. 8, 103-108 (2003).

3. C. N. Likos, Phys. Rep. 348, 267-439 (2001).

4. S. Kumar, M.-J. Lee, V. K. Aswal, and S.-M. Choi, Phys. Rev. E 87, 042315 (2013).

5. J. Kohlbrecher and W. Wagner, J. Appl. Crystallogr. 33, 804-806 (2000).

6. J. S. Pedersen, Adv. Colloid Interface Sci. 70, 171-210 (1997).

7. Y. Liu, W.-R. C hen, and S .-H. Chen, J. Chem. Phys. 122, 044507 (2005).

8. X. Ye, T. Narayanan, P. Tong, J. S. Huang, M. Y. Lin, B. L. Carvalho, and L. J. Fetters, Phys. Rev. E 54, 6500 (1996). 